\title{
Clinical profile of Relapse in Leprosy and Laboratory Correlation, including Mouse footpad inoculation and Molecular tests, in a Tertiary Leprosy Institution
}

\author{
Lakshmi Rajan $^{\mathrm{a}}$, Madhusmita Das ${ }^{\mathrm{b}}$, Joyce Ponnaiya ${ }^{\mathrm{c}}$ \& \\ Mannam Ebenezer ${ }^{\mathrm{d}}$

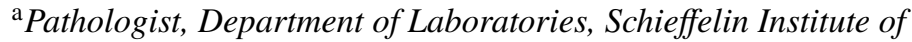 \\ Health-Research and Leprosy Center (SIH-R\&LC), Karigiri, Vellore, Tamil \\ Nadu - 632106, India \\ ${ }^{\mathrm{b}}$ Research Scientist, Molecular Biology and Immunology Division, \\ Schieffelin Institute of Health-Research and Leprosy Center (SIH-R\&LC), \\ Karigiri, Vellore, Tamil Nadu-632106, India \\ ${ }^{\mathrm{c}}$ Consultant Pathologist, Department of Laboratories, Schieffelin Institute of \\ Health-Research and Leprosy Center (SIH-R\&LC), Karigiri, Vellore, Tamil \\ Nadu - 632106, India \\ ${ }^{\mathrm{d}}$ Head of Global Programmes, The Mission To End Leprosy, India
}

Submitted 15 March 2021; Accepted 5 August 2021

\begin{abstract}
Summary
Introduction According to many studies, the relapse rate in leprosy has come down to negligible levels after the introduction of multi-drug therapy (MDT) by WHO. However, a small number of patients have been known to relapse after release from treatment, even when there has been good compliance.

Methods and findings During 2009-2017 (8 years), 23 multibacillary relapse cases were diagnosed clinically at SIHR\&LC, and investigated further to confirm relapse; $95.6 \%$ of cases were positive by slit skin smear, $78.3 \%$ on histopathology and $63.2 \%$ through growth in the mouse foot pad (MFP).

Conclusion The positive slit skin smear and the presence of active Hansen's disease with solid stained $M$. leprae on histopathology are suggestive of relapse. Positive mouse footpad inoculation indicated the presence of viable $M$. leprae. Relapse was confirmed in 12 patients, but negative mouse footpad results cannot exclude relapse. Where there are no facilities for such investigations, we must develop awareness about possible relapse in all treated patients, especially in field conditions, for early selfreporting, referral, and retreatment (with alternative drugs in cases of drug resistance) as needed.
\end{abstract}

Keywords: Relapse, Mycobacterium leprae, leprosy, mouse foot pad 


\section{Introduction}

Leprosy is a chronic bacterial infection involving skin and peripheral nerves. It is treated with multi-drug therapy (MDT) for six months in cases of paucibacillary (PB) leprosy and twelve months in multibacillary (MB) leprosy. The efficacy of MDT is measured by the number of relapses seen after completion of MDT and is expressed as the relapse rate. ${ }^{1}$

Relapse is defined as the occurrence of new skin lesions after completion of a standard course of MDT, or by an increase of two or more logs in the Bacillary Index (BI) compared with the previous BI at any one skin smear site. ${ }^{2}$ Since relapses usually occur years after completion of MDT, it is difficult to suspect and identify them under field conditions. ${ }^{3}$ Clinically, it is often difficult to differentiate between relapse and late reversal reaction.

Relapse is often attributed to either persistence of $M$. leprae in a dormant phase even after MDT, or drug-resistant mutants. ${ }^{4} \mathrm{~A}$ difference has been drawn between reactivation and relapse. While relapse occurs after complete and sustained subsidence of the disease, reactivation occurs mainly due to inadequate treatment. Similarly, a difference has been drawn between relapse and re-infection. Re-infection indicates lesions caused by new, exogenous infection after the subsidence of the disease, whereas relapse is usually caused by endogenous bacilli. ${ }^{5}$ It has been noted that patients with an initially high BI, who are treated with 12 doses of WHO MB MDT, are at a higher risk of relapse. ${ }^{6-8}$

A clinical diagnosis of relapse is made based on the appearance of new lesions. This is confirmed by slit skin smear examination and where possible by histological examination of a skin biopsy from a new lesion.

This study describes the clinical, microbiological, and histological features of MB relapse seen in a tertiary leprosy institution.

\section{Methods}

The criteria to diagnose relapse in this study included the occurrence of new skin lesions after completion of a standard course of MDT, or an increase of two or more logs in the Bacillary Index (BI) compared with the previous BI at any one skin smear site.

In this retrospective study, 23 multibacillary leprosy patients who took MB MDT previously were diagnosed as relapse cases at the Schieffelin Institute of Health - Research Leprosy Centre (SIH-RLC), Karigiri, India during 2009-2017, were included. Twenty-two patients presented with new skin lesions and one patient with an increase in $\mathrm{BI}>2+$ at one site and a Morphological Index (MI) of 2\%. Among these 23 patients, 21 were men and the ages ranged from 19 to 76 years. A detailed history was obtained, and all patients underwent complete clinical examination, followed by further investigations to confirm relapse. An informed and written consent for participation was obtained from all the subjects enrolled in the study following the ethical guidelines laid down by the Indian Council of Medical Research (ICMR). All the procedures conducted in the study were in accordance with the institutional ethical committee and with the 1964 Helsinki declaration and its later amendments or comparable ethical standards.

Disease activity was assessed basing on the presence of solid stained M. leprae. The BI of granulomas was graded according to the Ridley-Jopling scale. The presence of active granulomas was indicated by sheets of granulomatous inflammation with macrophages containing eosinophilic granular cytoplasm or finely foamy cytoplasm with solid stained M. leprae. Dermal nerve involvement was indicated by Schwann cell proliferation with solid stained M. leprae. Granuloma fraction $>10$ to $20 \%$ approximately. 
Histologically inactive Hansen's disease was identified by the following features: small collections of macrophages around blood vessels and neurovascular bundles; macrophages containing vacuolated cytoplasm and fragmented/granular forms of $M$. leprae; dermal nerves with perineural fibrosis and hyalinization; and perivascular chronic inflammation.

\section{SLIT SKIN SMEAR}

Slit skin smears were taken from all 23 relapse cases. The skin smear results were expressed as Bacillary Index (BI) and graded according to Ridley's logarithmic scale.

\section{SKIN BIOPSY}

Skin biopsies were taken from new skin lesions in 22 relapse cases and in one patient from the site where the BI had increased. After fixation, the tissue was processed in an automatic tissue processor; $5 \mu \mathrm{m}$ thick sections were cut using a rotary microtome with disposable blades. The sections were stained with hematoxylin and eosin for cellular details and with Ziehl Neelsen's stain for acid-fast M. leprae.

\section{MOUSE FOOT PAD INOCULATION FOR VIABILITY STUDIES}

Using thymectomized and irradiated (T900r) CBA mice, MFP inoculation was done to demonstrate $M$. leprae multiplication in 19 out of 23 relapse cases. With fresh biopsy material from an active lesion, the inoculum was prepared as a homogenous solution with a concentration of $10^{4} \mathrm{AFB} / 0.03 \mathrm{ml}$; a volume of $0.03 \mathrm{ml}$ was injected into the hind footpads of the mice. M. leprae multiplication was checked at 6 months, 9 months, and 12 months.

\section{MOUSE FOOT PAD INOCULATION STUDY FOR DRUG RESISTANCE}

Simultaneously, using another set of normal mice, drug resistance study was done for 19 relapse cases for all 3 drugs used in MB MDT. MFP inoculation was not done for 4 patients, as fresh tissue samples were not available at the time of the study.

\section{MOLECULAR DETECTION OF DRUG RESISTANCE}

\section{$P C R$ and Detection of Mutations within rpoB and folP gene}

DNA was extracted from the skin biopsies using a lysis protocol. WHO guidelines for the Sentinel Surveillance Study of Drug Resistance in Leprosy were employed for detecting mutations. Briefly, a $20 \mu \mathrm{l}$ PCR reaction mix was prepared using $10 \mu 1$ of Hot start PCR Master mix (Qiagen Inc. Netherlands), $2 \mu \mathrm{l}$ of Q solution (Qiagen Inc. Netherlands), $=2 \mu \mathrm{g}$ of DNA and forward and reverse primers for rpoB gene, each at $0.25 \mu \mathrm{M}$ concentration. The mixture was cycled 37 times on a thermal cycler at $94{ }^{\circ} \mathrm{C}$ for $1 \mathrm{~min}, 60^{\circ} \mathrm{C}$ for $1 \mathrm{~min}$, and $70{ }^{\circ} \mathrm{C}$ for 1 min which was preceded by initial denaturation at $95^{\circ} \mathrm{C}$ for $15 \mathrm{~min}$ and terminated by a final extension at $72{ }^{\circ} \mathrm{C}$ for $10 \mathrm{~min}$. A 3-5 $\mu 1$ of the amplified PCR product was electrophoresed on $2 \%$ agarose gel for detection of Folp amplicon. The presence of mutations within the amplified product was confirmed by DNA sequencing through a commercial agency. Sequence data were analyzed using MEGA Version -7 (Molecular Evolutionary Genetics Analysis) and Sequencer V. 5.4.6. 
Table 1. RJ classification at diagnosis and at relapse

\begin{tabular}{lcccc}
\hline RJ Classification & LL & BL & BT & Total \\
\hline At initial diagnosis & $16(69.6 \%)$ & $7(30.4 \%)$ & - & 23 \\
At relapse & $14(60.8 \%)$ & $8(34.8 \%)$ & $1(4.2 \%)$ & 23 \\
\hline
\end{tabular}

Table 2. Clinical presentation with smear results at relapse

\begin{tabular}{lcccccccc}
\hline Clinical presentation & \multicolumn{7}{c}{ Skin smear (BI) } \\
\cline { 2 - 8 } & $6+$ & $5+$ & $4+$ & $3+$ & $2+$ & $1+$ & Negative & Total \\
\hline Infiltration or nodules & 1 & 5 & 3 & 1 & - & 1 & - & 11 \\
Raised erythematous patches & - & - & 2 & 1 & 1 & - & - & 4 \\
Hypopigmented patches & - & - & - & 1 & - & - & 1 & 2 \\
ENL reaction & - & 3 & 1 & - & - & - & - & 4 \\
T1R reaction & - & 1 & - & - & - & - & - & 1 \\
BI is increased >2+ and MI 2\% & - & - & 1 & - & - & - & - & 1 \\
Total & 1 & 9 & 7 & 3 & 1 & 1 & 1 & 23 \\
\hline
\end{tabular}

\section{Results}

At the Schieffelin Institute of Health - Research Leprosy Centre, Karigiri, a tertiary leprosy referral center in South India, between 2009-2017, 22 patients presented with new skin lesions and one patient with an increase in $\mathrm{BI}>2+$ at one site and MI $2 \%$.

The duration between completion of MDT and the occurrence of clinical relapse ranged from one year to 25 years with an average of 9.71 years. 15 patients $(68.2 \%)$ relapsed after an interval of 6 years or more from completion of treatment.

Infiltration and/or nodules were the commonest lesions, seen in 11 patients (47.8\%), 4 patients $(17.4 \%)$ presented with erythematous raised patches, 4 patients $(17.4 \%)$ with ENL reaction, 2 patients $(8.7 \%)$ with hypopigmented patches, 1 patient $(4.3 \%)$ with Type 1 reaction, and 1 patient $(4.3 \%)$ with an increase in $\mathrm{BI}>2+$ two years after completing MDT.

At clinical diagnosis of relapse, 14 patients (60.8\%) were classified as lepromatous leprosy according to the Ridley Jopling classification, 8 patients (34.8\%) as borderline lepromatous (BL), and 1 patient (4.2\%) as borderline tuberculoid (BT). Most of the patients at relapse remained in the same category of RJ classification as at initial diagnosis. One LL patient relapsed as BL, one BL patient relapsed as LL, and one LL patient relapsed as BT (Table 1).

\section{SLIT SKIN SMEAR TEST}

Slit Skin Smears were done at first diagnosis, after completion of treatment, and at the time of relapse for 13 patients, who took their initial treatment at SIHR\&LC. Among these patients, the average $\mathrm{BI}(3.77+)$ at relapse was higher than the average BI (3+) at initial diagnosis. The remaining 10 patients, who took their initial MDT in other hospitals, reported to SIHR\&LC, Karigiri when they developed new lesions i.e. at the time of relapse.

The initial and post-treatment skin smear results were not available for these patients, as most of them relapsed after an interval of 10 years or more.

Slit Skin Smear results were positive in twenty-two patients $(95.6 \%)$ at relapse. Seventeen of these were above BI 4+ (74\%). Patients who presented with infiltration and/or nodules showed the highest BI values when compared to other clinical presentations (Table 2). 
Table 3. Clinical presentation compared with histopathology at relapse

\begin{tabular}{lccc}
\hline Clinical presentation & \multicolumn{2}{c}{ Histopathology } & \multirow{2}{*}{ Total } \\
\cline { 2 - 3 } & Active & Inactive & 11 \\
\hline Infiltration and or nodules & 8 & 3 & 4 \\
Raised erythematous patches/plaques & 3 & 1 & 2 \\
Hypopigmented patches & 2 & 1 & 4 \\
ENL reaction & 3 & - & 1 \\
Type 1 reaction & 1 & - & 1 \\
BI is increased 2+\& MI 1\% and 2\% at two sites & 1 & $5(21.7 \%)$ & $23(100 \%)$ \\
Total & $18(78.3 \%)$ & & \\
\hline
\end{tabular}

\section{SKIN BIOPSY RESULTS}

Histopathology was done for all 23 cases; active BL/LL leprosy histopathology with solid stained M. leprae was found in 17 cases. In 1 LL patient, histopathology showed smearnegative, active BT leprosy. Thus 18 cases have shown histopathologically active leprosy out of 23 patients. In one patient who relapsed as histoid leprosy, the macrophages were spindle-shaped with solid stained M. leprae. And one LL case who relapsed as BT had active epithelioid cell granulomas and dermal nerve involvement. Histopathology of the remaining five cases showed inactive BL/LL type of leprosy with fragmented/granular forms of M. leprae.

\section{Histopathology of co-existence of relapse and reaction}

In three patients, the skin biopsy showed ENL pathology—vasculitis, neutrophils, haemorrhage, intercellular oedema in the background of active BL/LL HD with solid stained M. leprae. In another patient, the skin biopsy showed Type 1 reaction pathology in the background of active BL HD with solid stained $M$. leprae. In one LL patient who was clinically diagnosed as a relapse with ENL reaction, the skin biopsy showed ENL pathology in the background of inactive LL HD with fragmented/granular forms of M. leprae. Though this patient was diagnosed as having both relapse and reaction clinically, histopathologically it was only an ENL reaction, without relapse.

The comparison between clinical features at relapse and disease activity seen in histopathology is shown in Table 3. There was a significant difference seen when the clinical presentation was compared with an active and inactive form of the disease $(p<0.0001)$. The clinical presentations were inclined towards a more active form of the disease in most of the relapse cases.

\section{MOUSE FOOT PAD (MFP) INOCULATION STUDY RESULTS}

Mouse Foot Pad (MFP) inoculation was done with fresh tissue from active skin lesions for 19 out of 23 relapse patients, inoculum prepared and injected into hind footpads. For 4 patients MFP was not done as fresh tissue was not available at the time of study. Growth was noticed as a positive result in 12 patients $(63.2 \%)$. There was no growth in 7 patients. When the smear status at relapse was compared with the MFP results, 10 out of 12 patients who showed growth in MFP had BI values 4+ or above.

There was no correlation between the growth of bacilli in the MFP and reaction status, as late reactions are common with dead bacilli without relapse. In this study 11 out of 19 patients had MFP +ve growth, but only 4 patients presented with reactions (3 ENL + 1 T1R). 
Table 4. Histopathology at relapse compared with MFP inoculation

\begin{tabular}{lccccc}
\hline Histopathology & Total & \multicolumn{4}{c}{ MFP Inoculation } \\
\cline { 3 - 5 } & & Done & Positive & Negative & Not done \\
\hline Active HD & 18 & 15 & 11 & 4 & 3 \\
Inactive HD & 5 & 4 & 1 & 3 & 1 \\
Total & 23 & 19 & 12 & 7 & 4 \\
\hline
\end{tabular}

Table 5. Comparison of drug resistance between Mouse foot pad and molecular method

\begin{tabular}{lcccccc}
\hline Method & $\begin{array}{c}\text { Sensitive to } \\
\text { DDS/rifampicin }\end{array}$ & $\begin{array}{c}\text { DDS } \\
\text { resistance }\end{array}$ & $\begin{array}{c}\text { Rifampicin } \\
\text { resistance }\end{array}$ & $\begin{array}{c}\text { No growth in MFP } \\
\text { (no viable bacilli) }\end{array}$ & $\begin{array}{c}\text { Test not } \\
\text { done }\end{array}$ & Total \\
\hline Molecular method & 17 & 4 & 1 & NA & 1 & 23 \\
MFP & 9 & 2 & 1 & 7 & 4 & 23 \\
\hline
\end{tabular}

\section{DRUG RESISTANCE STUDY RESULTS WITH MFP}

Simultaneously using another set of normal mice, drug resistance studies were done for 19 out of 23 relapse cases for all three drugs used in MB MDT.

Nine relapse cases were sensitive to all 3 drugs used in MBMDT. One relapse case was found to be resistant to rifampicin. This relapse patient was also found resistant to rifampicin by the molecular method. Two relapse cases were found to be resistant to DDS. Another 2 DDS resistant cases were identified by the molecular method, but the MFP was not done as the samples were not received. The rifampicin-resistant patient relapsed after an interval of 10 years. Two DDS-resistant patients relapsed after 21 years, one after 10 years and one after only one year.

Mouse Foot Pad inoculation results were also compared with the histopathology results at relapse. Out of 18 patients who showed active disease with solid stained $M$. leprae, MFP was done for 15 patients. 11 out of 15 cases $(73.3 \%)$ showed MFP growth. There was a significant difference $(p<0.0001)$ in positivity in the MFP with the active disease when compared to inactive cases (Table 4).

\section{DRUG RESISTANCE THROUGH MOLECULAR METHOD}

Drug Resistance through molecular methods was done for 22 out of 23 of the relapse cases. 17 patients were sensitive to all drugs. DDS resistance was seen in 4 patients. Rifampicin resistance was found in 1 patient and this patient was also resistant to rifampicin in the MFP study. Among the 4 DDS resistance cases, 2 showed mutation at the 53-codon position where Threonine is changed to Arginine in the folP gene (Thr53Arg) and 2 cases showed mutation at the 55 codon position where Proline is changed to Leucine (Pro55Leu) in the folP gene. In the patient with rifampicin resistance, the mutation was at the 441 position where Aspartate is changed to Tyrosine in the rpoB gene (Asp441Tyr).

The molecular method and MFP technique showed 100\% concordance in the results of the one patient with rifampicin and the two patients with DDS resistance. Of the 23 cases, 17 were seen to be sensitive by the molecular method and 9 by the MFP technique, however, 7 patients had no viable bacilli to further validate the drug resistance through MFP (Table 5). 


\section{Discussion}

The study describes the clinical features of relapse and their correlation with the Bacillary Index, histopathological findings, Mouse Foot Pad inoculation study, and Molecular testing for drug resistance among a cohort of multibacillary leprosy patients.

In this study relapse presented clinically at an average of 9.41 years after completion of MDT which is similar to other studies on MB relapse. ${ }^{9}$ Even though the period between completion of MDT and the appearance of new lesions ranged from 2 to 25 years, in nearly $70 \%$ of patients the gap was more than 6 years. MB relapses may occur after two years of completion of treatment at the earliest. However, a majority of MB relapses are likely to occur much later after RFT as seen in this study and other studies. Pattyn et al suggested that relapse resulting from inadequate treatment (MB case treated as $\mathrm{PB}$ ) leading to the poor killing of bacilli, is likely to result in early-onset relapse, while relapse resulting from persisting bacteria or drug-resistant mutants is likely to present as late-onset relapse. ${ }^{10}$

Twenty-two of these relapse patients self-reported to the health facility about the appearance of new lesions after completion of treatment. Self-reporting by patients should be encouraged as a strategy for detecting relapse early, as health programs may not have the necessary resources and personnel for long periods of surveillance to detect relapse cases of leprosy in the field.

Most relapses in this study (97\%) presented clinically at relapse with the same Ridley Jopling classification as at the initial diagnosis, indicating that the anergic status among these patients remained the same even after treatment. This finding is confirmed by the histopathological results and by other authors. ${ }^{9}$

$97 \%$ of clinically diagnosed relapses were confirmed by positive skin smears in this study. Skin smear examination is probably the most important tool to confirm a diagnosis of relapse. Even if previous skin smear results are not available, a positive skin smear result together with clinical features of new lesions should be considered as a confirmation of relapse.

Since it is known that the BI would fall at the rate of about one log per year, a BI result of $4+$ at initial diagnosis should have been reduced to at least $2+$ after two years of MDT. So, a BI result of 4+ or above at relapse two years after RFT can be considered significant in the diagnosis of relapse, even if initial skin smear results are not available.

Higher average skin smear value (3.77) at relapse, when compared to average skin smears value (3.00) at initial diagnosis as well as a shift of initial skin smear values towards the higher end of the BI scale at relapse, probably indicate that the immunological status of these patients for leprosy has not changed after treatment.

Histopathology findings confirmed the presence of active disease in $78.2 \%$ of clinically diagnosed, smear-positive relapse cases. Histopathological examination of skin provides useful insights to diagnose relapses such as the presence of solid bacilli, disease activity, disease resolution, and evidence of reactions. It is also very useful where available, to differentiate between reactions and active disease.

Confirmed growth in the Mouse Foot Pad supported the diagnosis of relapse in about $63 \%$ of clinically diagnosed, smear-positive relapses cases. $M$. leprae multiplication is not seen in patients with a low BI nor in patients with inactive HD, in which the histopathology shows mostly fragmented bacilli.

Molecular testing for identifying drug resistance among these relapses showed only one patient to have rifampicin resistance. DDS resistance which is already known and prevalent was seen in four patients. In our study, we showed that the molecular method and MFP technique showed $100 \%$ concordance in the results of the one patient for rifampicin and the two patients 
for DDS resistance. And out of the 23 cases, 17 were seen to be sensitive in molecular method and 9 in MFP technique, however, 7 patients had no viable bacilli to further validate the drug resistance through MFP. Hence, it is imperative to do both molecular and MFP tests wherever possible, to have a better understanding of how the bacteria works in vivo.

Most of the relapses seen in this study are of late-onset, the most likely cause being persisting M. leprae. Five cases are presented with evidence of drug resistance.

This study showed good concordance between clinical features and skin smear results for the diagnosis of relapse. Thus, skin smear examination is especially important in confirming a clinical diagnosis of relapse. Histopathology and Mouse Foot Pad inoculation showed a lower degree of concordance with a clinical diagnosis of relapse. Histopathology where available is useful especially in differentiating between disease activity and a reaction. Mouse Foot Pad test has a limited role in diagnosis and management of relapse as its results are available only after several months. Molecular method testing for drug resistance is useful in ruling out or confirming drug resistance as a cause for relapse. However, molecular methods need further research to identify additional codon sites for rifampicin resistance (if any) and a method to identify clofazimine resistance.

Health education to patients at RFT, to equip them to suspect and self-report in case of appearance of new lesions can be strengthened further. This will help in detecting relapse early by the patients themselves as health services for relapse surveillance may not be in place, or feasible or not optimal. The relapses can be treated early so that further transmission of the disease does not take place. Picture leaflets depicting the common presentations of new lesions as relapse can be given to the patent at RFT, with a session of health education.

In conclusion, the need for regular and adequate treatment with MDT for multibacillary patients cannot be overemphasized as the only way to prevent relapses. Regular MDT compliance will also minimize drug resistance. Since it will be difficult to prevent relapses caused by persisting organisms, drug resistance, and re-infection, the patient must be empowered to report the appearance of new lesions or any change in the status of old lesions to detect relapse early. Clinical and laboratory skills to diagnose relapses should be imparted to the key health professionals through capacity building. Strategic placement of equipped health centers in each district under the national programs will enable the diagnosis and treatment of relapses.

\section{Acknowledgements}

The authors would like to thank all the administrative and laboratory staff at "Schieffelin Institute of Health-Research and Leprosy Center - Karigiri," who were involved in sample collection and analysis of data. Our special thanks to Dr. Mannam who helped in the statistical analysis.

\section{Conflict of interest}

The authors declare that they do not have any conflict of interests.

\section{References}

1 Unit WHOL. Risk of relapse in leprosy. 1994 [cited 2021 Mar 5]; Available from: https://apps.who.int/iris/han dle/10665/61868.

2 Jamet P, Ji B. Relapse after long-term follow up of multibacillary patients treated by WHO multidrug regimen. Marchoux Chemotherapy Study Group. Int J Lepr Mycobact Dis Off Organ Int Lepr Assoc, 1995; 63(2): 195201. 
3 Waters MF. Relapse following various types of multidrug therapy in multibacillary leprosy. Lepr Rev, 1995; 66(1): 1-9.

4 Jerajani HR. Book review. Indian J Dermatol Venereol Leprol, 2010; 76(3): 309-310.

5 Ramu G. Clinical features and diagnosis of relapses in leprosy. Indian J Lepr, 1995; 67(1): 45-59.

6 Cellona RV, Balagon MFV, dela Cruz EC, Burgos JA, Abalos RM, Walsh GP et al. Long-term efficacy of 2 year WHO multiple drug therapy (MDT) in multibacillary (MB) leprosy patients. Int J Lepr Mycobact Dis Off Organ Int Lepr Assoc, 2003; 71(4): 308-319.

7 Girdhar BK, Girdhar A, Kumar A. Relapses in multibacillary leprosy patients: effect of length of therapy. Lepr Rev, 2000; 71(2): 144-153.

8 Jamet P, Ji B. Relapse after long-term follow up of multibacillary patients treated by WHO multidrug regimen. Marchoux Chemotherapy Study Group. Int J Lepr Mycobact Dis Off Organ Int Lepr Assoc, 1995; 63(2): 195201.

9 Shetty VP, Wakade AV, Ghate SD, Pai VV, Ganapati RR, Antia NH. Clinical, histopathological and bacteriological study of 52 referral MB cases relapsing after MDT. Lepr Rev, 2005; 76(3): 241-252.

10 Pattyn SR, Groenen G, Bourland J, De Muynck A, Grillone S, Grossetete G et al. The incubation time of relapses after treatment of multibacillary leprosy with rifampicin containing regimens. Eur J Epidemiol, 1988; 4(2): $231-234$.

11 Desikan KV. Relapse, reactivation or reinfection? Indian J Lepr, 1995; 67(1): 3-11. 